\title{
NONLINEAR RESET INTEGRATOR CONTROL DESIGN: APPLICATION TO THE ACTIVE SUSPENSION CONTROL OF VEHICLES
}

\author{
Leonardo Acho \\ Universitat Politènica de Catalunya (UPC) \\ Escola Universitària d'Enginyeria Tècnica Industrial de Barcelona(EUETIB), \\ Department of Applied Mathematics-III (MA-III), \\ and CoDAlab (http://www.ma3.upc.edu/codalab/). \\ Comte d'Urgell, 187, 08036, Barcelona, Spain. \\ Email:leonardo.acho@upc.edu
}

\begin{abstract}
We present an unexampled reset integrator control design based on the Clegg integrator system. Using an appropriate mathematical model of our Clegg integrator controller, stability proof of the closed-loop system applied to the vibration control problem of a second-order system is shown without invoking hybrid system theory. Furthermore, we illustrate the applicability of our controller, from the numerical experiment point of view, to the suspension vibration control of vehicles.
\end{abstract}

\section{KEY WORDS}

Reset control, stability, suspension control, vehicles.

\section{Introduction}

The first reset integrator controller was proposed by Clegg in 1958 to improve transient response of closed-loop linear systems [1]. This controller was proposed from the electronics point of view. Basically, the Clegg integrator controller is a linear system that resets to zero when its input is equal to zero. The main advantage of this reset behavior of the Clegg integrator is the reduction of overshoot caused by a class of integrator controllers, like the PI and PID ones (see [1], [2], [3], [4], and references there in). Although there exist mathematical models of the Clegg integrator system (see, for instance, [5], [6], and [7]); here, we propose a new mathematical representation of the Clegg integrator useful to prove stability of closed-loop system almost straightforwardly using a smooth Lyapunov function instead of invoking hybrid systems theory. Furthermore, utilizing this alternative representation, we propose a new Clegg integrator controller utilizable to the suspension control problem of vehicles.

On the other hand, car suspension control has the objective to increase the dynamic performance of vehicles, like stability and comfort (see, for instance, [8], [9], and references there in). Basically, there exist three types of suspension control of cars: 1) passive, 2) active, and 3) semi-active ([10]). Each one with theirs corresponding advantages and disadvantages. Here, we are interested in the active control one. Also, for control design and testing, we use the quarter car model due to its simplicity and because this model has been, for a long time, the par excellence model utilized to design and test car suspension control ([10]).

The rest of the paper is structured as follows. Section two shows the Clegg integrator controller and describing it using our mathematical model approach. Section three states a modification of the Clegg integrator controller. Some numerical experiments applied to the vibration control a second-order system are illustrated to highlight our controller performance. Stability proof of the equilibrium point of the closed-loop system is stated too by invoking Lyapunov theory and LaSalle's principle. Section four gives numerical experiments of the proposed controller applied to the suspension control of vehicles by appealing the quart car model. Finally, Section five states the conclusions.

\section{Reset integrator control design}

The original Clegg integrator control circuit is shown in Figure 1 [1]. In this figure, $R_{d}$ is very small compared to $R$. These resistances $\left(R_{d}^{\prime} s\right)$ are used to prevent short-circuit on the diodes and they are ignored for the control law design [1]. The output resistances $R_{s}$ are used to produce a summing node from the operational amplifier outputs [1]. Applying circuit theory to the upper operational amplifier (Fig. 1), we obtain:

$$
E_{o 1}=\left\{\begin{array}{rc}
-\beta \int E_{i} d t, & E_{i}>0 \\
0, & E_{i} \leq 0,
\end{array}\right.
$$

And for the bottom one, we have:

$$
E_{o 2}=\left\{\begin{array}{rc}
-\beta \int E_{i} d t, & E_{i}<0 \\
0, & E_{i} \geq 0
\end{array}\right.
$$

The parameter $\beta=1 / R C$ is the integrator gain. Combining (1) and (2) (due to the summing node previously commented), we obtain: 


$$
E_{o}=E_{o 1}+E_{o 2}=-\beta\left(\int\left|E_{i}\right| d t\right) \operatorname{sgn}\left(E_{i}\right),
$$

where

$$
\operatorname{sgn}(x)=\left\{\begin{array}{rc}
1, & x>0 \\
0, & x=0 \\
-1, & x<0
\end{array}\right.
$$



Figure 1. Clegg integrator circuit controller.

The representation of the Clegg controller given in (3)-(4) is an alternative mathematical model different from those stated, for instance, in [6] and [5]. Actually, and because of the signum function $\operatorname{sgn}(\cdot)$, this controller belongs to the chattering class (see, for instance, [12]).

\section{Reset control integrator design for second- order linear systems}

\subsection{Control design}

Consider the general second-order linear system:

$$
\ddot{x}+2 \zeta w_{n} \dot{x}+w_{n}^{2} x=w_{n}^{2} u,
$$

where $\zeta$ is the damping ration, $w_{n}$ is the undamping natural frequency, and $u$ is the control input. The standard regulation control objective consists to find a control law $u$ such that:

$$
\lim _{t \rightarrow \infty}(x, \dot{x})=(0,0) .
$$

Next is our main result.
Theorem 1.- Given the control law:

$$
\begin{aligned}
& u=-k_{i} y \operatorname{sgn}(\dot{x}), \\
& \dot{y}=|\dot{x}|,
\end{aligned}
$$

with a positive constant value $k_{i}$. Then, the equilibrium point $(x, \dot{x})=(0,0)$ of closed-loop system (5) and (7) is globally asymptotic stable. Moreover, $y(t)$ remains bounded.

Proof.- Consider the next Lyapunov function:

$$
V:=V(x, \dot{x}, y ; t)=\frac{w_{n}^{2} x^{2}}{2}+\frac{\dot{x}^{2}}{2}+\frac{k_{i} w_{n}^{2} y^{2}}{2} .
$$

Then, its time derivative along of the trajectories of the closed-loop system, yields:

$$
\dot{V}=-2 \zeta w_{n} \dot{x}^{2} .
$$

By invoking LaSalle's principle, we conclude that the equilibrium point of the closed-loop system $((x, \dot{x})=(0,0))$ is globally asymptotic stable. And we also conclude that $\dot{y}=0$ meaning that $y(t)$ remains bounded (on time).

Remark 1.-Observe that an alternative representation of (7) is:

$$
u=-k_{i}\left(\int|\dot{x}| d t\right) \operatorname{sgn}(\dot{x}) .
$$

\subsection{Numerical experiments}

Consider the system (5) with $\zeta=0.1, w_{n}=1$, and for the controller (7), $k_{i}=1$. Simulation results are shown in Figure 2. Figure 3 shows the corresponding phase portrait whereas Figure 4 gives the associate control law. The signum function approximation used was:

$$
\operatorname{sgn}(\dot{x}) \approx \frac{\dot{x}}{|\dot{x}|+0.01} .
$$

Observing Figures 2 and 3, we can appreciate that the controlled system shows no damping oscillation (as it was anticipated by the Clegg controller system theory).

\section{The active suspension control problem}

The active suspension control problem of vehicles consists to design a control law to improve stability performance by reducing vibrations in terms of safety, comfort, and durability (Chaper 9, [11]). From the control point of view, this is an interesting problem due to the stiffness and damping system matrices are no symmetric [11].

Consider an active vehicle suspension system described in Figure 5. Its dynamic's motion is [11]: 


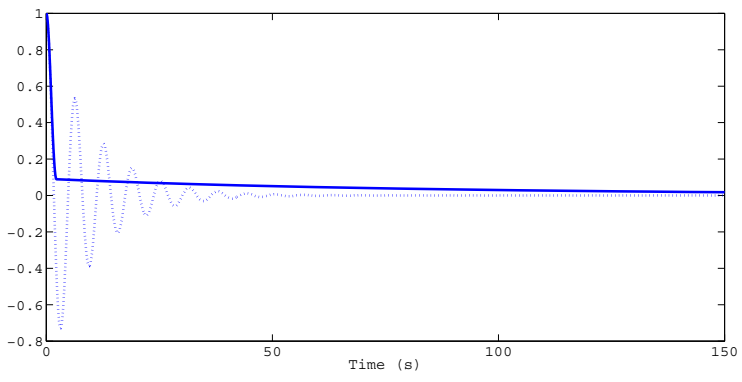

Figure 2. Simulation results $(x(t)$ versus time): dottedlines is the uncontrolled case $(u=0)$ and the solid one is the controlled scenario (initial conditions: $x(0)=1$, $\dot{x}(0)=0$, and $y(0)=0)$.

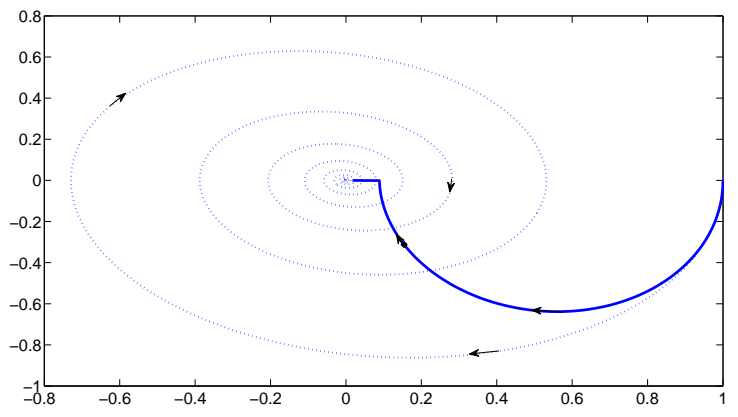

Figure 3. Simulation results (phase portrait: $\dot{x}(t)$ versus $x(t)$ ): dotted-lines is the uncontrolled case $(u=0)$ and the solid one is the controlled scenario.



Figure 4. Control law.

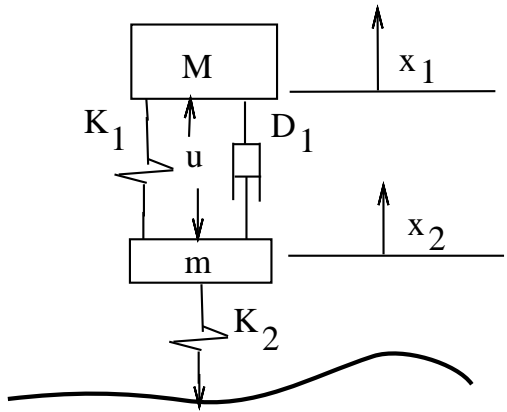

Figure 5. An active suspension system of a vehicle (a quarter car model).

$$
\begin{aligned}
M \ddot{x}_{1} & =u-K_{1}\left(x_{1}-x_{2}\right)-D\left(\dot{x_{1}}-\dot{x_{2}}\right) \\
m \ddot{x}_{2} & =-u+K_{1}\left(x_{1}-x_{2}\right)+D\left(\dot{x}_{1}-\dot{x}_{2}\right) \\
& -K_{2} x_{2}
\end{aligned}
$$

where $M=3000 \mathrm{~kg}$ represents the vehicle body mass, $m=500 \mathrm{~kg}$ is the unsprung part of the vehicle (including tyres and axles). $K_{1}=3000 \mathrm{~N} / \mathrm{m}$ and $K_{2}=30000 \mathrm{~N} / \mathrm{m}$ are the mechanical stiffness of the suspension system. $D=500 \mathrm{~N} / \mathrm{ms}^{-1}$ is the dashpot. Finally, $u$ is the active suspension control force in Newton units.

Let us design our integrator controller using the same thinking that we use to design a traditional PID-controller. That is, we design it without any acknowledge of the plant but adjusting the controller parameters carefully [13]. So, our controller would be:

$$
\begin{aligned}
u & =-k_{i} y \operatorname{sgn}\left(\dot{x}_{1}-\dot{x}_{2}\right), \\
\dot{y} & =\left|\dot{x}_{1}-\dot{x}_{2}\right| .
\end{aligned}
$$

Then, we only have a parameter to tune. Using $k_{i}=50$, simulation results are shown in Figure 6. Figure 7 gives the corresponding control law. Moreover, we used the next realization of the signum function:

$$
\operatorname{sgn}\left(\dot{x}_{1}-\dot{x}_{2}\right) \approx \frac{\dot{x}_{1}-\dot{x}_{2}}{\left|\dot{x}_{1}-\dot{x}_{2}\right|+0.01} .
$$

From Figure 6, we can perceive that the car controlled suspension system utilizing our Clegg controller, $x_{1}(t)$ displays almost no damping oscillation. This damping elimination is so desirable in vibration control of vehicles [10], [8], [9].

\section{Conclusion}

We have proposed a reset integrator controller by invoking the Clegg integrator system. According to this theory, this 

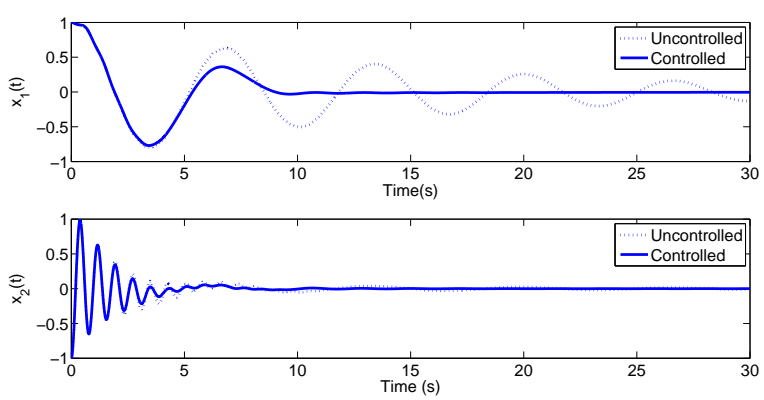

Figure 6. Numerical experiment results (initial conditions: $x_{1}(0)=1, x_{2}(0)=-1$, and $\left.\dot{x}_{1}(0)=\dot{x}_{2}(0)=y(0)=0\right)$.



Figure 7. Control law.

controller can be easily realized using analog electronic circuits via operational amplifiers. From the numerical point of view, we could appreciate that this kind of reset controllers can be employed to the vibration suspension control of vehicles. Moreover, we have granted a mathematical model of our reset controller useful to test stability of a closed-loop system.

As a future work, and observing that we have used a smooth approximation of the signum function (loosing some performance property of discontinuous-chattering controllers [12]), with operational amplifier circuits, this signum function realization would be almost exactly to the desired one. So, real experimental results using the reset integrator realization with analog electronics would be profitable (in mind with the possibility of a patent).

Finally, we have an open problem: stability proof of the proposed controller to the car suspension control.

\section{Acknowledgement}

This work was supported by the Spanish Ministry of Economy and Competitiveness under grant DPI201232375/FEDER.

\section{References}

[1] J. Clegg, A nonlinear integrator for servomechanisms, American Institute of Electrical Engineers, 77, 1958, 41-42.

[2] Y. Guo, Y.Wang, L. Xie, \& J. Zheng, Stability analysis and design of reset systems: Theory and an application, Automatica, 45, 2009, 492-497.

[3] O. Beker, C. V. Hollot, \& Y. Chait, Plant with integrator: an example of reset control overcoming limitations of liner feedback, IEEE Trans. on Automatic Control, 46(11), 2001, 1797-1799.

[4] Y. Zheng, Y. Chait, C. V. Hollot, M. Steinbuch, \& M. Norg, Experimental demonstration of reset control design, Control Engineering Practice, 8, 2000, 113120.

[5] L. Zaccarian, D. Nešić, \& A. R. Teel, Proc. American Control Conference, Portland, OR, 2005, 563-568.

[6] D. Nešić, L. Zaccarian, \& A. R. Teel, Stability properties of reset systems, Automatica, 44, 2008, 20192026.

[7] J. Carrasco, A. Baos, \& A. van der Schaft, A passivity-based approach to reset control systems stability, Systems \& Control Letters, 59, 2010, 18-24.

[8] K. Spentzas, S. A. Kanarachos, Design of a non-linear hybrid car suspension system using neural networks, Mathematics and Computers in Simulation, 60 , 2002, 369-378.

[9] S. Türkay, H. Akçay, Aspects of achievable performance for quater-car active suspensions, Journal of Sound and Vibration, 311 , 2008, 440-460.

[10] Guglielmino, E., Sireteanu, T., Stammers, C. W., Ghita, G., and Giuclea, M. , Semi-active suspension control: Improved vehicle ride and road friendliness (London, Springer, 2008).

[11] F. Lin, Robust control design: an optimal control approach (England, John Wiley \& Sons, Ltd, 2007).

[12] Y. Orlov, J. Alvarez, L. Acho, \& L. Aguilar. Global position regulation of friction manipulators via switched chattering control, International Journal of Control, 76(14), pp. 1146-1452, 2003.

[13] G. F. Franklin, J. D. Powell, \& A. E. Naeini, Feedback control of dynamic systems (N.Y., Addison Wesley, 2nd ed., 1991). 